\title{
Management of Bile Leak After Laparoscopic Hydatid Liver Surgery
}

\author{
Walid Faraj, MD, Eliane Malek, MD, Najla Itani, MD, Abdallah Abou El Naaj, MA, \\ Ghina El Nounou, MS, Fadi El Merhi, MD, Ali Haidar, MD, Mohamad Khalife, MD \\ Department of Surgery, (Drs. Faraj, Malek, Itani, Mr. El Naaj, Ms. El Nouno, Dr. Khalife), Department of Radiology, \\ (Drs. El Merhi, Haidar), American University of Beirut Medical Center, Beirut, Lebanon.
}

\begin{abstract}
Introduction: The liver is the organ most commonly affected by hydatid disease and is involved in up to $70 \%$ of cases. Surgical management can be open or laparoscopic; it can vary from a radical procedure such as hepatic resection or pericystectomy or a more conservative approach such as partial cystectomy. Bile leak after surgery can occur in between $13 \%$ and $26 \%$ of cases.

Case Description: We present the case of a 47-year-old patient who was diagnosed with 2 large hydatid liver cysts in segments V and VI and segments II and III, respectively. She underwent laparoscopic pericystectomy for both cysts and was discharged 2 days later. She presented after 10 days complaining of abdominal pain and fever. Computed tomography scan of the abdomen showed a $6 \times 7-\mathrm{cm}$ collection at the surgical site of segments II and III. A percutaneous drain was inserted and drained $60 \mathrm{~mL}$ of dark bilious fluid. The patient did well after drainage and was discharged home after 6 days.

Discussion: Irrespective of the surgical treatment chosen, bile leaks may complicate any procedure and must always be considered seriously. Although there have been numerous studies concerning bile leak after open surgery, there are only a few describing bile leaks after laparoscopic surgery. Managing bile leaks after hydatid cyst excision is still a matter of debate. Some physicians would advocate conservative management; other physicians would recommend a further procedure such as sphincterotomy or biliary stenting after endoscopic retrograde cholangiopancreatography.
\end{abstract}

Key Words: Hydatid disease, Open surgery, Laparoscopic surgery, Bile leak.

Citation Faraj W, Malek E, Itani N, El Naaj AA, El Nounou G, El Merhi F, Haidar A, Khalife M. Management of bile leak after laparoscopic hydatid liver surgery. CRSLS e2014.00060. DOI: 10.4293/CRSLS.2014.00060.

Copyright (C) 2014 SLS This is an open-access article distributed under the terms of the Creative Commons Attribution-Noncommercial-ShareAlike 3.0 Unported license, which permits unrestricted noncommercial use, distribution, and reproduction in any medium, provided the original author and source are credited.

Address correspondence to: Walid Faraj, MD, Department of Surgery, Liver Transplantation and HBP Unit, American University of Beirut Medical Center, PO Box: 11-0236, Riad El Solh, Beirut 1107 2020, Beirut, Lebanon. Telephone: +9613969610; Fax: +9611364961; E-mail: wf07@aub.edu.lb

\section{INTRODUCTION}

As an endemic health problem in the Mediterranean countries and the Middle East and an increasingly global issue because of immigration, hydatid disease is a pertinent entity that many physicians must tackle. The liver is the most commonly affected organ and is involved in up to $70 \%$ of patients. ${ }^{1,2}$ Hence open surgical treatment, be it a radical procedure such as hepatic resection or pericystectomy or a more conservative approach such as partial cystectomy, may precipitate several biliary complications. Many studies have shown that bile leaks occur in anywhere between $13 \%$ and $26 \%$ of patients undergoing an open procedure. ${ }^{2-4}$ However, there is a paucity of studies examining the significance of bile leak after the advent of radical or conservative laparoscopic removal of hydatid cysts. Furthermore, a review of the proper management of such complications is necessary. We present the case of a 47-year-old female patient who underwent a complicated laparoscopic hydatid liver surgical procedure.

\section{CASE REPORT}

A 47-year-old female patient presented with epigastric pain of a few days' duration. Computed tomography (CT) scan and ultrasonography of the abdomen showed a $7 \times$ 9-cm hydatid cyst in segments V and VI of the liver and 
a $5 \times 6-\mathrm{cm}$ complex hydatid cyst in segments II and III (Figure 1).

Laparoscopic partial pericystectomy was performed for both cysts. Intraoperatively, a large biliary fistula was identified in the left cyst and sutured, and 2 drains were inserted. The patient was started on a regimen of albendazole postoperatively and was discharged home on day 4 postoperatively. Two weeks later, the patient presented to the emergency department of our institution with a fever, chills, and a dry cough of a few hours' duration. On physical examination, the patient was found to have decreased breath sounds in the right and left lower lobes and epigastric tenderness. The patient's white blood cell count was 23100 with a left shift of 93\%. The findings of urinalysis and blood and urine cultures were negative. A chest film confirmed suspected pneumonia. CT scan of the abdomen showed a $6 \times 7-\mathrm{cm}$ collection at the surgical site of segments II and III. A percutaneous drain was inserted and drained $60 \mathrm{~mL}$ of dark bilious fluid. The patient started receiving intravenous antibiotics. The plan was to perform endoscopic retrograde cholangiopancreatography (ERCP) if the patient's condition did not improve. However, 2 days later, the white blood cell count had decreased to 13300 and the patient was afebrile. The drainage decreased over a few days and then it dried up. The patient was discharged home in excellent condition. Follow-up CT scan showed complete resolution of the cysts (Figure 2).

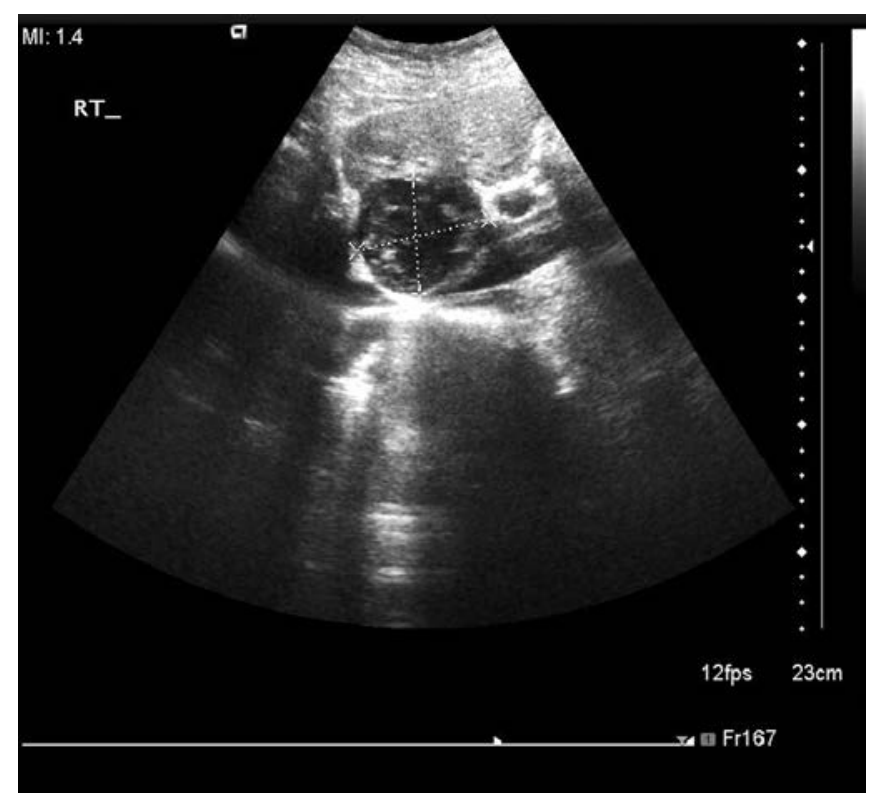

Figure 1. Ultrasonography of liver showing hydatid cysts.

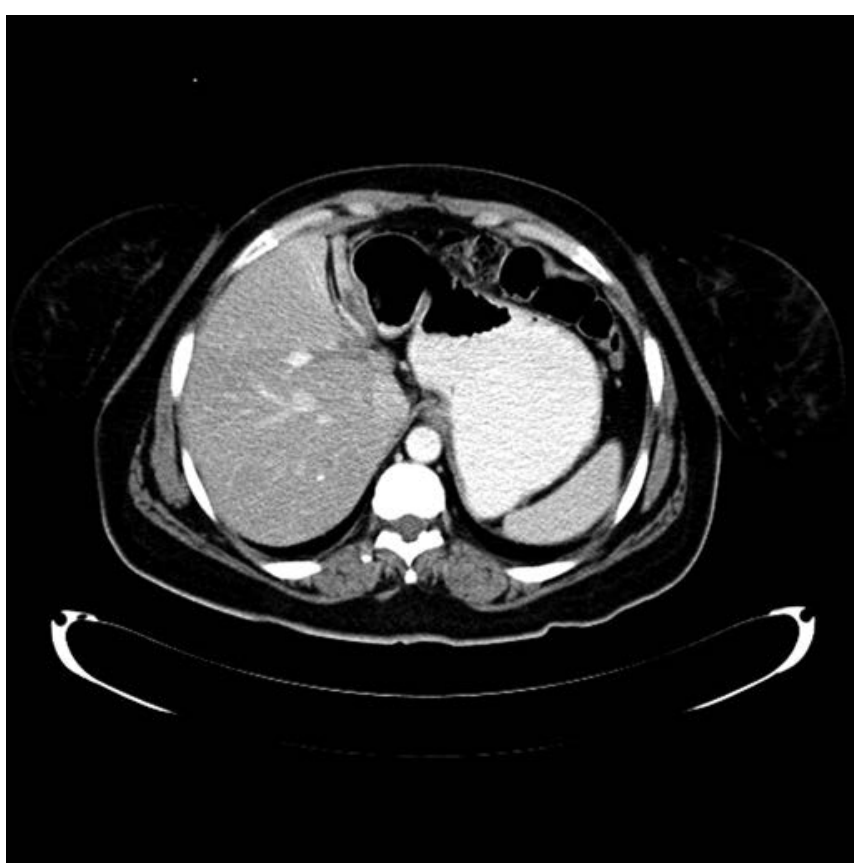

Figure 2. CT scan of liver showing complete resolution of cysts.

\section{DISCUSSION}

Although surgery has remained the first line of treatment for hepatic hydatid disease, there is still a great deal of controversy over which procedure to choose. The surgical options are numerous, but overall, they can be placed into 1 of 2 categories: open and larparoscopic. Regarding open surgery, several recent studies have shown that the radical approach is associated with lower rates of recurrence, fewer complications such as bile leak, and decreased mortality rates. ${ }^{5-7}$ However, some physicians will still argue that radical surgery may carry a serious perioperative risk in a patient with benign disease and will opt for the conservative approach. ${ }^{3}$

Most surgical procedures for hepatic hydatid disease performed by an open approach can be performed laparoscopically. Laparoscopic surgery offers the known advantages of minimal invasiveness, reduction in hospital stay, and decreased rate of wound complications. Furthermore, when the surgeon is using a laparoscope, the ability to examine the details of the cavity of the cyst enables him or her to detect cystobiliary communications with enhanced accuracy. ${ }^{8}$ Studies have shown consistently positive results that reinforce the use of this technique. Busic et al. ${ }^{9}$ performed laparoscopic surgery in 2 groups of patients (1 group underwent a conservative procedure and the other group, radical) and performed open surgery in another 2 
similar groups. No complications or recurrences occurred within both laparoscopic groups. Yagci et al ${ }^{10}$ compared open, laparoscopic, and percutaneous approaches. There were 2 postoperative deaths in the open surgery group, and bile leak occurred in 28 patients, 10 patients, and 2 patients after open surgery, percutaneous treatment, and laparoscopic treatment, respectively. Perhaps the lower rate of complications with hydatid laparoscopic procedures is because of better visualization of the cystobiliary communications that can then be managed intraoperatively and not cause further complications postoperatively. Overall, laparoscopic surgery is a safe alternative to open surgery, if not the preferred method. ${ }^{8-12}$

Many studies have designated the importance of selection criteria that aid in determining whether an open or laparoscopic approach is better. Cyst location can assist one in establishing this. Cysts located centrally carry a higher risk of bleeding, and an open approach has been recommended in such cases; cysts located in segments VI and VII are not safe for laparoscopic access. ${ }^{7,8}$ Moreover, selection of radical versus conservative open surgery is based on patient characteristics, cyst anatomy, and surgical expertise. ${ }^{6}$ Therefore perhaps the same should apply when deciding whether to perform a radical versus conservative laparoscopic procedure. As for cyst content, it has been recommended that complicated cysts be addressed by radical procedures, in contrast to uncomplicated cysts, which may be better handled conservatively. ${ }^{7,13}$

With respect to the case presented, it is important to note that according to the previously described recommendations, an open technique would have been preferred for the cyst located in segments V and VI and radical removal would have been ideal for the complicated cyst in segments II and III. However, a laparoscopic conservative procedure was performed instead (partial pericystectomy). It is very reasonable that had the aforementioned criteria been adopted, the bile leak may have been prevented altogether. However, it is important to note that successful laparoscopic resection of the cyst involving segments $\mathrm{V}$ and $\mathrm{VI}$ took place, even if segment $\mathrm{V}$ was centrally located and segment VI may not be considered a segment with safe access. Therefore the location of the cyst should not be considered a clear-cut determinant of the type of surgery to proceed with, and further studies are needed to assess this determinant.

Irrespective of the surgical treatment chosen, bile leaks may complicate any liver surgery. Although there have been numerous studies concerning bile leak after open surgery, there are only a few describing bile leaks after laparoscopic surgery. Khoury et al ${ }^{14}$ reported on $12 \mathrm{pa}-$ tients treated by a laparoscopic approach, and only 1 patient had bile leak that ceased spontaneously. These findings were confirmed by Ertem et al, ${ }^{15}$ who reported bile leak in 1 patient after conservative laparoscopic surgery. These results show great promise for laparoscopic techniques, but further randomized controlled trials are necessary to compare laparoscopic and open techniques regarding bile leak.

Bile leaks can be caused by communicating ducts opening into the residual cavity, thereby causing cystobiliary communications. This was noticed in our patient intraoperatively, and suturing was performed. However, the bile leak occurred postoperatively, indicating the possible existence of smaller cystobiliary communications that may have been missed during the operation. Although laparoscopy offers the advantage of visualizing the cystic cavity with greater detail, this case suggests that some occult communications missed intraoperatively may still present as postoperative bile leaks. Demircan et al ${ }^{3}$ designated preoperative clinical predictors of occult cystobiliary communications presenting as postoperative bile leaks. Among the clinical predictors were an alkaline phosphatase level $>250 \mathrm{U} / \mathrm{L}$, total bilirubin level $>17.1 \mu \mathrm{mol} / \mathrm{L}$, direct bilirubin level $>6.8 \mu \mathrm{mol} / \mathrm{L}, \gamma$-glutamyl transferase level $>34.5 \mathrm{U} / \mathrm{L}$, eosinophil count $>0.09$, and cyst diameter $>8.5 \mathrm{~cm}$. Regardless of the fact that these conclusions were based on a study in which open surgery was performed, the same procedures can be performed laparoscopically. Hence, one can attempt to use the same predictors of bile leak after open surgery for bile leak after laparoscopic surgery. Regarding our patient, the alkaline phosphatase level was 77 , the $\gamma$-glutamyl transferase level was 108 , the total bilirubin level was 0.3 , and the direct bilirubin level was 0.2. Hence, if these predictors had been used, a bile leak should not have occurred. Therefore further studies are needed to evaluate the cutoff values for predictors of bile leak in laparoscopic surgery. These predictors can then be combined with the criteria mentioned earlier for the selection of an open versus laparoscopic procedure to help establish a basis on which preliminary decisions regarding management can be made.

Management of postoperative bile leaks is variable. ERCP was performed to localize the leak in addition to verifying the appearance of bile in the percutaneous drainage of abdominal collections. ${ }^{1}$ Furthermore, procedures such as sphincterotomy or biliary stenting after ERCP were carried out to resolve the bile leaks. Other studies have shown that a more conservative approach is preferred because it was 
found that most bile leaks spontaneously resolve, particularly if a low output is noted., ${ }^{2,4,13}$ Hence the decision to perform ERCP and possible sphincterotomy or biliary stenting can be determined by a quantitative characterization of the bile leak. After confirmation of the diagnosis of a bile leak and subsequent biloma, our patient started receiving antibiotics and her condition was monitored. Because the drain placed was characterized by a low output $(25 \mathrm{~mL})$, our patient's condition was conservatively monitored with the belief that her symptoms would resolve and the bile leak would spontaneously cease.

Certain guidelines must be adopted and standardized for the management of bile leaks after hydatid cyst removal. Perhaps one can follow an algorithm designed for the management of bile leaks after laparoscopic cholecystectomy because the causes of bile leak in these cases mirror the causes of bile leak after hydatid cyst removal. Ahmad et al ${ }^{16}$ proposed that if an effective drain is left in situ during the operation and is draining bile, initiation of antibiotics and daily assessment of drain output are all that are needed. It was shown that drainage of $200 \mathrm{~mL}$ of bile per day or less and reduction of this amount over a period of a few days indicate that the leak is likely to subside on its own. It is only when peritonitis, sepsis, or $>200 \mathrm{~mL}$ of bile drain per day is found that invasive interventions must be considered. ${ }^{16}$

\section{References:}

1. Agarwal N, Sharma BC, Garg S, et al. Endoscopic management of postoperative bile leaks. Hepatobiliary Pancreat Dis Int 2006;5:273-277.

2. Agarwal S, Sikora SS, Kumar A, et al. Bile leaks following surgery for hepatic hydatid disease. Indian J Gastroenterol 2005; $24: 55-58$.

3. Demircan O, Baymus M, Seydaoglu G, et al. Occult cystobiliary communication presenting as postoperative biliary leakage after hydatid liver surgery: are there significant preoperative clinical predictors? Can J Surg 2006;49:177-184.

4. Gahukamble DB, Khamage AS, Gahukamble LD. Outcome of minimal surgery for hydatid cysts of the liver in children with reference to post-operative biliary leakage. Ann Trop Paediatr 2000;20:147-151.

5. De Werra C, Condurro S, Tramontano S, et al. Hydatid disease of the liver: thirty years of surgical experience. Chir Ital 2007;59:611-625.

6. Priego P, Nuno J, Hervas PL, et al. Hepatic hydatidosis. Radical vs. conservative surgery: 22 years of experience. Rev Esp Enferm Dig 2008;100:82-85.

7. Sayek I, Tirnaksiz MB, Dogan R. Cystic hydatid disease: current trends in diagnosis and management. Surg Today 2004; 34:987-996.

8. Chen W, Xusheng L. Laparoscopic surgical techniques in patients with hepatic hydatid cyst. Am J Surg 2007;194:243-247.

9. Busic Z, Lemac D, Stipancic I, et al. Surgical treatment of liver echinococcosis-the role of laparoscopy. Acta Chir Belg 2006; 106:688-691.

10. Yagci G, Ustunsoz B, Kaymakcioglu N, et al. Results of surgical, laparoscopic, and percutaneous treatment for hydatid disease of the liver: 10 years experience with 355 patients. World J Surg 2005;29:1670-1679.

11. Chowbey PK, Shah S, Khullar R, et al. Minimal access surgery for hydatid cyst disease: laparoscopic, thoracoscopic, and retroperitoneoscopic approach. J Laparoendosc Adv Surg Tech A 2003;13:159-165.

12. Polat FR, Polat S, Sultanoglu E. Laparoscopic treatment of hydatid cyst of the liver: a case report. JSLS 2005;9:245-246.

13. Kayaalp C, Bzeizi K, Demirba AE, et al. Biliary complications after hydatid liver surgery: incidence and risk factors. J Gastrointest Surg 2002;6:706-712.

14. Khoury G, Jabbour-Khoury S, Bikhazi K. Results of laparoscopic treatment of hydatid cysts of the liver. Surg Endosc 1996; 10:57-59.

15. Ertem M, Uras C, Karahasanoglu T, et al. Laparoscopic approach to hepatic hydatid disease. Dig Surg 1998;15:333-336.

16. Ahmad F, Saunders RN, Lloyd GM, et al. An algorithm for the management of bile leak following laparoscopic cholecystectomy. Ann R Coll Surg Engl 2007;89:51-56. 